\title{
Effective theory for ultracold strongly interacting fermionic atoms in two dimensions
}

\author{
Fan $\mathrm{Wu}^{1}$ * Jianshen $\mathrm{Hu}^{1}$ * Lianyi $\mathrm{He}^{1,2}$, Xia-Ji Liu ${ }^{3}$, and $\mathrm{Hui} \mathrm{Hu}^{3}$ \\ ${ }^{1}$ Department of Physics and State Key Laboratory of Low-Dimensional \\ Quantum Physics, Tsinghua University, Beijing 100084, China \\ ${ }^{2}$ Collaborative Innovation Center of Quantum Matter, Beijing 100084, China and \\ ${ }^{3}$ Centre for Quantum and Optical Science, Swinburne University of Technology, Melbourne, Victoria 3122, Australia
}

(Dated: April 16, 2020)

\begin{abstract}
We propose a minimal theoretical model for the description of a two-dimensional (2D) strongly interacting Fermi gas confined transversely in a tight harmonic potential, and present accurate predictions for its zero-temperature equation of state and breathing mode frequency based on existing auxiliary-field quantum Monte Carlo data. We show that the minimal model Hamiltonian needs at least two independent interaction parameters, the $2 \mathrm{D}$ scattering length and effective range of interactions, in order to quantitatively explain recent experimental measurements with ultracold 2D fermions. We resolve in a satisfactory way the puzzling experimental observations of the smaller than expected equations of state and breathing mode frequency. Our establishment of the minimal model for 2D fermions is crucial to understanding the Berezinskii-Kosterlitz-Thouless transition in the strongly correlated regime.
\end{abstract}

Two-dimensional (2D) quantum many-body systems are of great interest, due to the interplay of reduced dimensionality and strong correlation, which leads to enhanced quantum and thermal fluctuations [1] and a number of ensuing quantum phenomena such as Berezinskii-Kosterlitz-Thouless (BKT) physics [2, 3]. In this respect, the recently realized 2D Fermi gas of ultracold ${ }^{6} \mathrm{Li}$ and ${ }^{40} \mathrm{~K}$ atoms under a tight axial confinement provides a unique platform [4, 5], with unprecedented controllability particularly on interatomic interactions. To date, many interesting properties of ultracold 2D Fermi gases have been thoroughly experimentally explored [5], including the equation of state (EoS) at both zero temperature [6, 7] and finite temperature [8, 9], radio-frequency spectroscopy [10 12], pair momentum distribution [13], firstorder correlation function and BKT transition [14], and quantum anomaly in breathing mode frequency [15 17]. These results may shed light on understanding other important strongly correlated $2 \mathrm{D}$ systems, such as high- $T_{c}$ layered cuprate materials [18], ${ }^{3} \mathrm{He}$ submonolayers 19], exciton-polariton condensates [20] and neutron stars 21].

The present theoretical model of ultracold 2D Fermi gases is simple [4, 5]. Under a tight harmonic confinement with trapping frequency $\omega_{z}$ along the axial $z$-axis and a weak confinement $\omega_{\perp}$ in the transverse direction, the kinematic $2 \mathrm{D}$ regime is reached when the number of atoms $N$ is smaller than a threshold $N_{2 D} \simeq\left(\omega_{z} / \omega_{\perp}\right)^{2}$, so all the atoms are forced into the ground state of the motion along $z[5]$. The interatomic interactions are then described by a single $s$-wave scattering length $a_{2 D}[\underline{6}$, which is related to a 3D scattering length $a_{3 D}$ via the quasi-2D scattering amplitude 22]. Various experimental data have been compared and benchmarked with different theoretical predictions of the simple 2D model 23 32]. For EoS, i.e., the chemical potential and pressure at essentially zero temperature, good agreements were found [6, 9]. But, at the quantitative level the experi- mental data somehow lie systemically below the accurate predictions from auxiliary-field quantum Monte Carlo (AFQMC) simulations [6, 9]. The discrepancy is not so serious and might be viewed as an indicator of small deviation from the $2 \mathrm{D}$ kinematics [5], in spite of the fact that the $2 \mathrm{D}$ condition $N \ll N_{2 D}$ is well satisfied. However, a serious problem does arise when two experimental groups measured the breathing mode frequency in the deep 2D regime most recently [16, 17]. The observed frequency turned out to be much smaller than the well-established theoretical prediction in the strongly interacting regime 25, 26]. This discrepancy is at the qualitative level, suggesting that the simple 2D model with a single parameter $a_{2 D}$ may not be sufficient for the description of ultracold 2D Fermi gases 33.

The purpose of this Letter is to provide a minimal theory of ultracold 2D Fermi gases, with the inclusion of a properly defined effective range of interactions (see Fig. $1)$. The significant role played by effective range was realized in our previous work [33]. However, the determination of the effective range there turns out be problematic. We solve the proposed model Hamiltonian at zero temperature by taking into account strong pair fluctuations at Gaussian level and beyond (Fig. 2), with the help of a correlation energy from AFQMC in the zerorange limit [30]. This enables us to predict accurate EoS (Fig. 3 and Fig. 4), as well as reliable breathing mode frequency (Fig. 5). The puzzling quantitative and qualitative discrepancies, observed in the previous comparisons between experiment and theory [5, [6, 9, 16, 17], are therefore naturally resolved in a satisfactory way.

Effective range of interactions. We start by considering the collision of two fermions with mass $M$ and unlike spin in a highly anisotropic harmonic trapping potential, described by a quasi-2D scattering amplitude [22],

$$
f_{Q 2 D}\left(k ; a_{3 D}, a_{z}\right)=\frac{4 \pi}{\sqrt{2 \pi} a_{z} / a_{3 D}+\varpi\left(k^{2} a_{z}^{2} / 2\right)},
$$




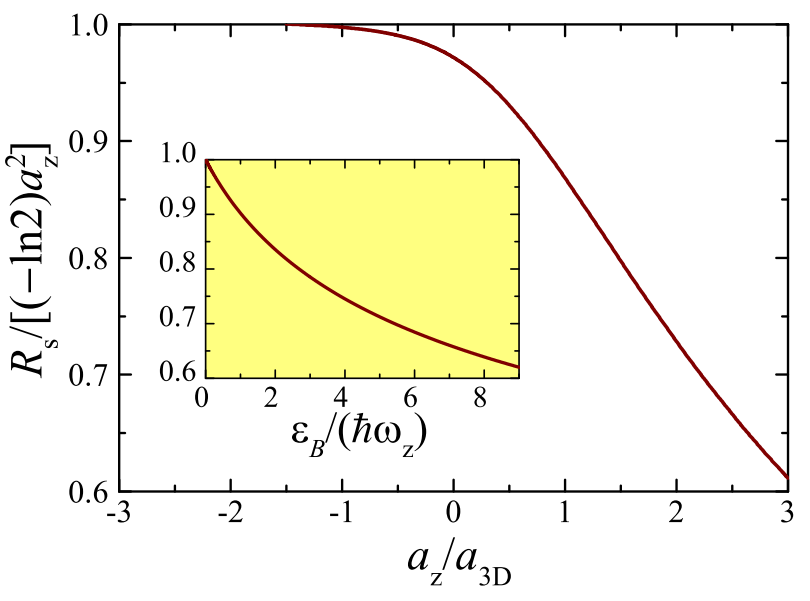

FIG. 1. Confinement-induced effective range of interactions $R_{s}$, in units of $R_{s}^{(0)}=(-\ln 2) a_{z}^{2}$, as a function of the inverse $3 \mathrm{D}$ scattering length $a_{z} / a_{3 D}$. The inset shows the effective range as a function of the two-body binding energy.

where $a_{z} \equiv \sqrt{\hbar /\left(M \omega_{z}\right)}$ is the harmonic oscillator length along the $z$-axis and the function $\varpi(x)$ has the expansion $\varpi(x \rightarrow 0) \simeq-\ln (2 \pi x / \mathcal{B})+(2 \ln 2) x+i \pi$ with $\mathcal{B} \simeq 0.905$ 22]. In the simplest treatment, one may parameterize the quasi-2D collision using a $2 \mathrm{D}$ scattering length $a_{2 D}$ [5, 6], by setting the 2D scattering amplitude $f_{2 D}\left(k ; a_{2 D}\right)=-2 \pi / \ln \left[k a_{2 D}(k) / i\right]=f_{Q 2 D}\left(k ; a_{3 D}, a_{z}\right)$. In general, one thus obtains a momentum-dependent $a_{2 D}(k)$, which in the zero-energy limit takes the form $a_{2 D}(k \rightarrow 0)=a_{s} \equiv a_{z} \sqrt{\pi / \mathcal{B}} \exp \left(-\sqrt{\pi / 2} a_{z} / a_{3 D}\right)$ 22]. The advantage of this simple treatment is that the description universally depends on a single parameter $a_{2 D}$, to be evaluated at a characteristic collision momentum $k_{0}$, i.e., $k_{0}=\sqrt{2 M \tilde{\mu}} / \hbar$, where $\tilde{\mu}$ is the chemical potential that does not include the two-body binding energy $[\underline{5}, \underline{6}]$.

A more adequate parametrization of the $2 \mathrm{D}$ collision is to include an effective range of interactions $R_{s}$ in the 2D scattering amplitude [35],

$$
f_{2 D}\left(k ; a_{s}, R_{s}\right)=\frac{4 \pi}{-2 \ln \left(k a_{s}\right)-R_{s} k^{2}+i \pi},
$$

whose pole gives a two-body bound state with binding energy $\varepsilon_{B}=\hbar^{2} \kappa^{2} / M$, where the wavevector $\kappa$ satisfies $R_{s}=2 \ln \left(\kappa a_{s}\right) / \kappa^{2}$. The same two-body bound state should be supported by the pole of the quasi-2D scattering amplitude in Eq. (10) as well. By setting $k \rightarrow i \kappa$ there, we find $\sqrt{2 \pi} a_{z} / a_{3 D}+\varpi\left[-\varepsilon_{B} /\left(2 \hbar \omega_{z}\right)\right]=0$ [36] . Therefore, we can directly calculate the effective range $R_{s}$, once $\varepsilon_{B}$ or $\kappa$ is solved at a given $a_{z} / a_{3 D}$.

The effective range obtained in this way is reported in Fig. 1 It decreases monotonically from $R_{s}^{(0)} \equiv(-\ln 2) a_{z}^{2}$ with increasing $a_{z} / a_{3 D}$ (main figure) or binding energy $\varepsilon_{B}$ (inset). We note that $R_{s}^{(0)}$ can be easily derived from the second expansion term in $\varpi(x \rightarrow 0)$ and its magni-

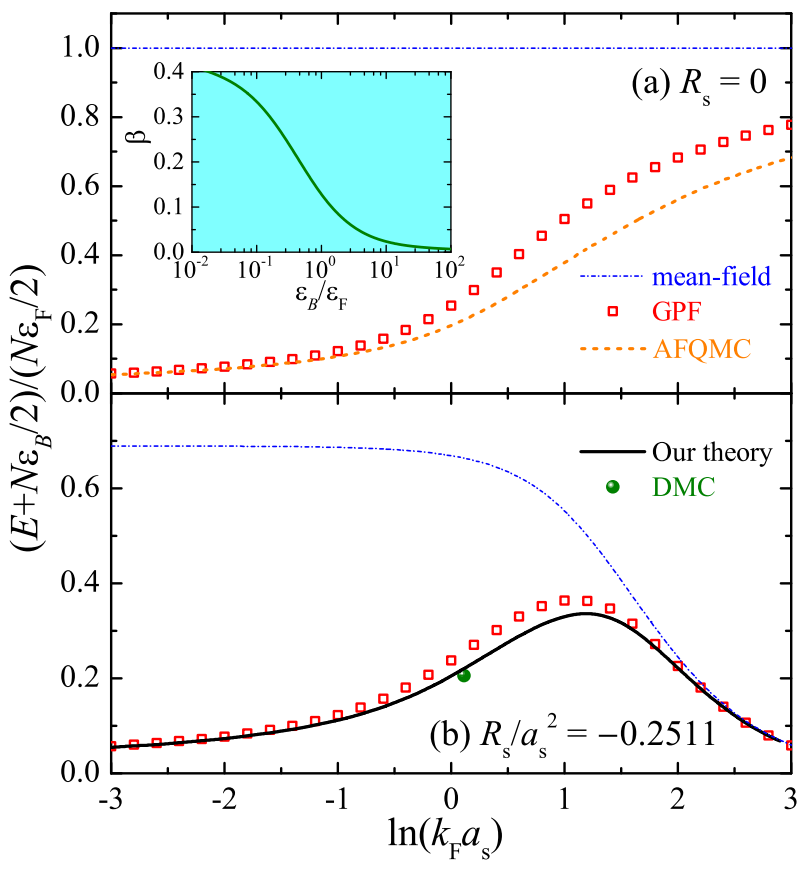

FIG. 2. Total energy with the two-body bound-state energy subtracted as a function of $\ln \left(k_{F} a_{s}\right)$, at $R_{s}=0$ (a) and $R_{s} \simeq-0.2511 a_{s}^{2}$ (b). The mean-field and GPF predictions are shown by blue dot-dashed lines and red squares, respectively. At zero range in (a), the latest AFQMC result 30] is plotted by orange dashed line. The inset shows the beta function $\beta=\Delta E_{\mathrm{c}} / \Delta E_{\mathrm{GPF}}$ (see Eq. (4)). At finite range in (b), our theory (black solid line) is compared with the DMC data (green dot) 38].

tude, i.e., $R_{s}^{(0)} \sim a_{z}^{2}$, is a clear indication of the quasi-2D nature of atom collisions [5, 22]. As the wavefunction of two colliding atoms at distance within $a_{z}$ is set by the full 3D contact interaction potential, these collisions can never be purely 2D. They can only be approximately treated as $2 \mathrm{D}$, out of the range $\sim a_{z}$.

Many-body theory. To account for the effective range $R_{s}$, it is useful to adopt a two-channel model [33, 37, 38]:

$$
\begin{aligned}
\mathcal{H}= & \sum_{\mathbf{k} \sigma=\{\uparrow, \downarrow\}} \xi_{\mathbf{k}} c_{\mathbf{k} \sigma}^{\dagger} c_{\mathbf{k} \sigma}+\sum_{\mathbf{q}}\left(2 \xi_{\mathbf{q} / 2}+\nu\right) b_{\mathbf{q}}^{\dagger} b_{\mathbf{q}} \\
& +\frac{g}{\sqrt{\mathcal{S}}} \sum_{k q}\left(b_{\mathbf{q}} c_{\mathbf{q} / 2+\mathbf{k} \uparrow}^{\dagger} c_{\mathbf{q} / 2-\mathbf{k} \downarrow}^{\dagger}+\text { h.c. }\right),
\end{aligned}
$$

where $\xi_{\mathbf{p}} \equiv \hbar^{2} \mathbf{p}^{2} /(2 M)-\mu$, and $c_{\mathbf{k} \sigma}$ and $b_{\mathbf{q}}$ are the annihilation operators of atoms and molecules in the openand closed-channel, respectively. The channel coupling $g$ is related to $R_{s}$, via $R_{s}=-4 \pi^{2} \hbar^{4} /\left(M^{2} g^{2}\right)$, the detuning $\nu$ of molecules is tuned to reproduce the binding energy $\varepsilon_{B}$, i.e., $\nu=-\varepsilon_{B}+\left(g^{2} / \mathcal{S}\right) \sum_{\mathbf{k}}\left[\hbar^{2} \mathbf{k}^{2} / M+\varepsilon_{B}\right]^{-1}[33,38]$, and $\mathcal{S}$ is the area.

We solve the model Hamiltonian at different orders of approximation at zero temperature. Formally, the ground-state energy $E$ may be decoupled as,

$$
E\left[\ln \left(k_{F} a_{s}\right), k_{F}^{2} R_{s}\right]=E_{\mathrm{MF}}+\Delta E_{\mathrm{GPF}}+\Delta E_{\mathrm{c}},
$$


where $k_{F}=(2 \pi n)^{1 / 2}$ is Fermi wavevector and $\varepsilon_{F}=$ $\hbar^{2} k_{F}^{2} /(2 M)$ is Fermi energy for a system with number density $n$. The mean-field (MF) theory provides the leading term $E_{\mathrm{MF}}$, while the major correction arising from strong pair fluctuations at Gaussian level can be obtained by using the Gaussian pair fluctuation (GPF) theory [29, 33, 39 41], i.e., $\Delta E_{\mathrm{GPF}}=E_{\mathrm{GPF}}-E_{\mathrm{MF}}$. The effect of pair fluctuations beyond Gaussian level may be characterized by a correlation energy $\Delta E_{\mathrm{c}}$, which is anticipated to be much smaller than $\Delta E_{\mathrm{GPF}}$. To see this, in Fig. 2(a) we plot the ground-state energy in the zerorange limit $\left(R_{S}=0\right)$, predicted by mean-field theory, GPF theory [29] and AFQMC simulation [30]. Indeed, the correlation energy given by the difference between the GPF and AFQMC energies is notably smaller than $\Delta E_{\mathrm{GPF}}$. In particular, $\Delta E_{\mathrm{c}}$ becomes vanishingly small in the tight-binding limit of $\ln \left(k_{F} a_{s}\right) \rightarrow-\infty$ [29]. It is then useful to define a beta function $\beta=\Delta E_{\mathrm{c}} / \Delta E_{\mathrm{GPF}} \ll 1$, which varies as functions of the two dimensionless interaction parameters $\ln \left(k_{F} a_{s}\right)$ and $k_{F}^{2} R_{s}$. For small $k_{F}^{2} R_{s}$, however, it seems plausible to assume that $\beta$ relies on $\varepsilon_{B} / \varepsilon_{F}$ only, whose dependence can be readily extracted in the zero-range limit using the AFQMC data, as shown in the inset of Fig. 2(a). Other possible choice of the $\beta$-function is considered in Supplemental Material [42].

We thus establish a viable procedure to calculate the ground-state energy at nonzero effective range. For a given set $\left(k_{F} a_{s}, k_{F}^{2} R_{s}\right)$, we first calculate the binding energy $\varepsilon_{B} / \varepsilon_{F}$ and determine the value of $\beta$. Both meanfield and GPF theories are then applied to obtain $E_{\mathrm{MF}}$ and $\Delta E_{\mathrm{GPF}}$, and consequently $\Delta E_{\mathrm{c}}=\beta \Delta E_{\mathrm{GPF}}$. In Fig. 2(b), we present $E=E_{\mathrm{GPF}}+\Delta E_{\mathrm{c}}$ in black line for a fixed ratio $R_{s} / a_{s}^{2} \simeq-0.2511$, at which we may benchmark our prediction against available high-precision diffusion Monte Carlo (DMC) data (i.e., the single green dot) [38, 43]. We find that the correction $\Delta E_{\mathrm{GPF}}$ becomes smaller at nonzero effective range. Towards the non-interacting limit $\left(a_{s} \rightarrow \infty\right)$ and hence large $k_{F}^{2} R_{s}$, $\Delta E_{\mathrm{GPF}}$ vanishes quickly. This is understandable, since pair fluctuations become weaker with decreasing channel coupling $g$ and even mean-field theory may provide accurate prediction at sufficiently large $k_{F}^{2} R_{s}[38]$. The correlation energy also significantly reduces at finite effective range and we find $\left|\Delta E_{\mathrm{c}}\right|<0.02 N \varepsilon_{F}$ at all interaction strengths for $R_{s} / a_{s}^{2} \simeq-0.2511$. The agreement between our theory with DMC is excellent, with a difference less than $0.01 N \varepsilon_{F}$.

Equation of state. Once the ground-state energy $E$ of a uniform 2D Fermi gas is determined, we calculate directly the chemical potential $\mu$ and pressure $P$ using standard thermodynamic relations. Experimentally, these homogeneous EoS can be extracted from a low-temperature trapped Fermi gas, by using the local density approximation [44], which assigns a local chemical potential $\mu(r)=\mu_{\text {peak }}-V(r)$ to each position $r$ in the potential $V(r)=M \omega_{\perp}^{2} r^{2} / 2$. Both the peak chemical potential

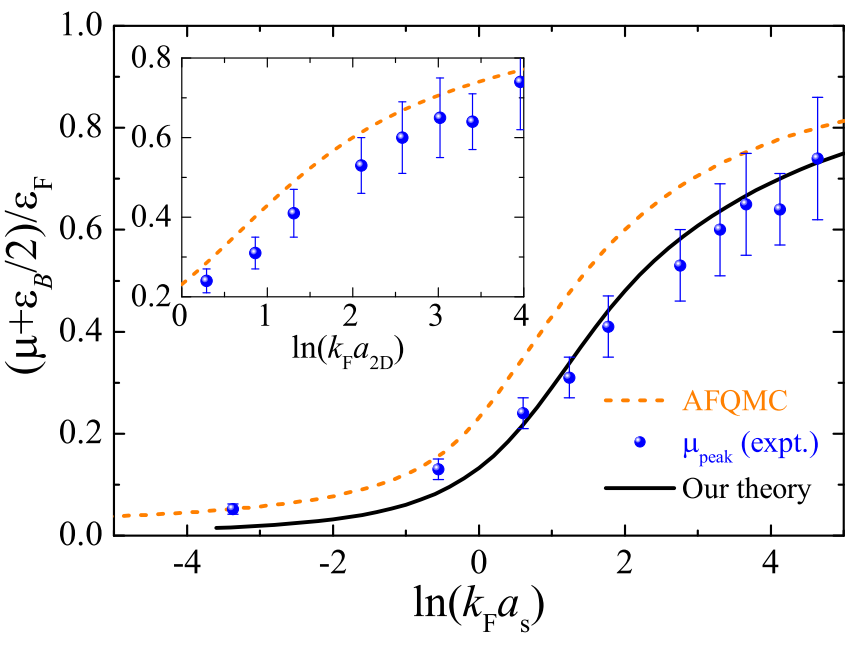

FIG. 3. Chemical potential with the two-body bound state contribution subtracted, as a function of $\ln \left(k_{F} a_{s}\right)$ at the number of atoms $N=N_{2 D}$. The predictions of AFQMC (i.e., for zero effective range) [30] and our theory with a realistic effective range as in the experiment [9] are shown by orange dashed line and black solid line, respectively, and are compared with the experimental data for $\mu_{\text {peak }}$ (blue circles) measured at $N \simeq N_{2 D}$ [9, 45]. The inset shows the chemical potential as a function of $\ln \left(k_{F} a_{2 D}\right)$, where $a_{2 D}$ is the effective scattering length adopted in the experiment [9].

$\mu_{\text {peak }}$ and the in situ density distribution $n(r)$ can be experimentally measured [6, [8, 9], from which one deduces the homogeneous density $\operatorname{EoS} n(\mu)$. By further using the force balance condition [6], $\nabla P(r)=-n(r) \nabla V(r)$, the homogeneous pressure $\operatorname{EoS} P(n)$ can also be determined.

In Fig. 3, we show the experimental data for the peak chemical potential $\mu_{\text {peak }}$, measured at different magnetic fields (i.e., $\left.a_{3 D}\right)$ and hence at different $\ln \left(k_{F} a_{s}\right)$ [9, 45]. Our predictions for the peak chemical potential, calculated under the same experimental condition, are plotted by the black solid line. We find a good agreement between theory and experiment at $\ln \left(k_{F} a_{s}\right)>0$. Due to the large effective range of interactions in the experiment (i.e., $k_{F}^{2} R_{s} \lesssim-1.2$ at $N \simeq N_{2 D}[9]$ ), the zero-range predictions from AFQMC appear to strongly over-estimate the chemical potential. The use of an effective $a_{2 D}$ can not fully explain the discrepancy (see the inset and also Fig. 1 in Ref. [9]), as we mentioned earlier. The discrepancy can also be hardly understood by possible systematic effects such as finite temperature and the failure of a 2D model due to a finite filling factor $N / N_{2 D}$ [42, 46].

In Fig. 4, we present the comparison between our predictions and the experimental data [6, 7] for pressure at the trap center. In this case, we have $N \simeq 0.35 N_{2 D}$ and therefore the effect of the effective range may become weaker. Nevertheless, we can see clearly that in the strongly interacting regime (i.e., $0<\ln \left(k_{F} a_{s}\right)<2$ ), the experimental data lie systematically below the zero-range results from AFQMC. The model Hamiltonian with a fi- 


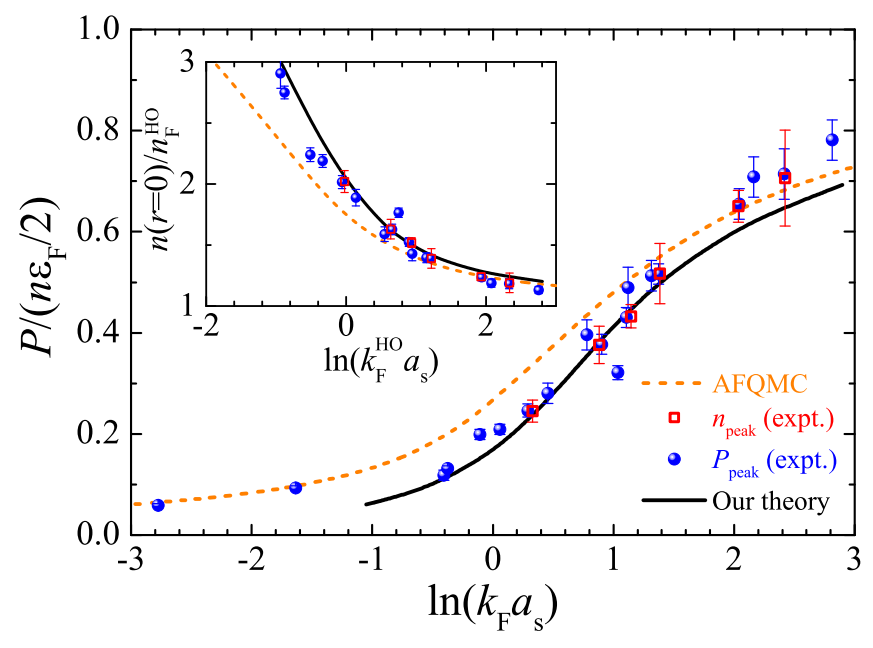

FIG. 4. Pressure as a function of $\ln \left(k_{F} a_{s}\right)$ at $N=0.35 N_{2 D}$. We use blue circles and red squares to show the experimental data from Ref. [6] and Ref. [7] with $N \simeq 0.35 N_{2 D}$, respectively. The predictions of AFQMC [30] at zero range and our theory at finite range are shown by orange dashed line and black solid line, respectively. Towards the weakly interacting limit, the finite-temperature effect may become sizable and up-shift the pressure data [6]. The inset shows the peak density (in units of $\left.n_{F}^{\mathrm{HO}}\right)$ as a function of $\ln \left(k_{F}^{\mathrm{HO}} a_{s}\right)$, where $n_{F}^{\mathrm{HO}}$ and $k_{F}^{\mathrm{HO}}=\left(2 \pi n_{F}^{\mathrm{HO}}\right)^{1 / 2}$ are the peak density and wave-vector of an ideal Fermi gas in traps.

nite effective range should be used, in order to quantitatively understand the experimental measurement. We note that, in harmonic traps the pressure at the center is fixed by the force balance condition to $P=M \omega_{\perp}^{2} N /(2 \pi)$ 7]. Using the peak density of an ideal trapped Fermi gas $n_{F}^{\mathrm{HO}}=M \omega_{\perp} \sqrt{N} /(\pi \hbar)$ [5], we find that the peak density $n \equiv n(r=0)$ can be written in terms of the pressure at the trap center, i.e., $n / n_{F}^{\mathrm{HO}}=\left[P /\left(n \varepsilon_{F} / 2\right)\right]^{-1 / 2}$. This provides an alternative way to illustrate the data, as shown in the inset.

In both Fig. 3 and Fig. 4 the agreement between theory and experiment becomes worse at small $k_{F} a_{s}$, suggesting the inadequacy of our theory towards the limit of a Bose-Einstein condensate (BEC). This is because, experimentally the BEC regime is reached by changing $a_{3 D}$ instead of $k_{F}$. For a small positive $a_{3 D}$ the system is better viewed as a quasi-2D weakly interacting BEC, with a $2 \mathrm{D}$ scattering length $a_{2 D}^{(m)}$ determined from the $3 \mathrm{D}$ molecular scattering length $a_{3 D}^{(m)} \simeq 0.6 a_{3 D}$ [47] and with an effective range $R_{s}^{(m)} \sim-a_{z}^{2}$. Our two-channel model cannot fully recover this interaction-driven BEC limit. For more details, we refer to Supplemental Material [42].

Breathing mode and quantum anomaly. We now turn to consider the breathing mode frequency, which was recently measured in two experiments at $N \simeq 0.2 N_{2 D}$ [16, 17], as shown in Fig. 5 by green circles and blue squares. Theoretically, the zero-temperature breathing mode frequency can be conveniently calculated by using

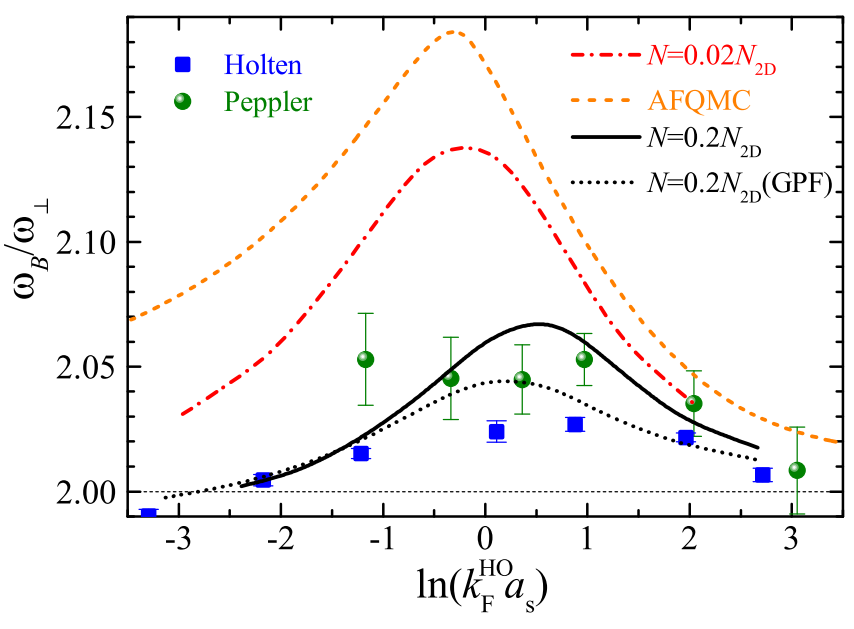

FIG. 5. Breathing mode frequency of $2 \mathrm{D}$ strongly interacting fermions as a function of the interaction parameter $\ln \left(k_{F}^{\mathrm{HO}} a_{s}\right)$, at different total number of atoms $N / N_{2 D} \rightarrow 0$ (AFQMC [52], orange dashed line), 0.02 (red dot-dashed line), and 0.2 (black solid line) as in two recent experiments by Holten et al. 16] at $0.10-0.18 T_{F}$ (green circles) and Peppler et al. 17] at $0.14-0.22 T_{F}$ (blue squares). Here $T_{F}$ is the Fermi temperature. We show also the GPF prediction in the dotted line.

the sum-rule approach [48, 49],

$$
\hbar^{2} \omega_{B}^{2}=-2\left\langle r^{2}\right\rangle\left[\frac{d\left\langle r^{2}\right\rangle}{d\left(\omega_{\perp}^{2}\right)}\right]^{-1},
$$

where $\left\langle r^{2}\right\rangle=N^{-1} \int d^{2} \boldsymbol{r}\left[r^{2} n(r)\right]$ is the squared radius of the Fermi cloud at a given trapping frequency $\omega_{\perp}$. In the classical treatment, a 2D Fermi gas is scale-invariant [50] and acquires a polytropic density $\operatorname{EoS}, \mu(n) \propto n^{2}$. As a result, the mode frequency is pinned to $2 \omega_{\perp}$, regardless of temperature and interactions [50]. The deviation of the breathing mode frequency away from $2 \omega_{\perp}$ can be viewed a quantum anomaly [25, 26], arising from strong quantum pair fluctuations in 2D [51].

As readily seen from Fig. 5, the observed quantum anomaly in the two experiments is far below the prediction from AFQMC for zero-range interactions with a single 2D scattering length [52]. It can only be understood when we use the proposed minimal model for 2D ultracold fermions and take into account the realistic finite effective range at $N \simeq 0.2 N_{2 D}$. The quantitative difference between our theory and experiment at $0<\ln \left(k_{F} a_{s}\right)<1$ could be caused by the finite temperature in the two experiments [42], which is in the range $[0.10-0.22] T_{F}$.

It turns out that the breathing mode frequency or quantum anomaly depends sensitively on the effective range. The zero-range result of AFQMC can hardly be asymptotically approached, even we decrease the number of atoms down to just a few percent of $N_{2 D}$ (see the red dot-dashed line at $N=0.02 N_{2 D}$ ). In this case, however, the deviation from the classical limit of $2 \omega_{\perp}$ is very signif- 
icant and its experimental confirmation will deepen our understanding of the long-sought 2D quantum anomaly in cold atoms [51], which was recently observed [53.

Conclusions. We have established a minimal model to describe ultracold interacting fermions confined in two dimensions and have solved it accurately at zero temperature with the help of existing AFQMC results. We have shown that the confinement-induced effective range of interactions has to be included, in order to understand the recent measurements on quantum anomaly in a qualitative manner and on equation of state at quantitative level. Our results pave the way to investigate the crucial role played by effective range in other two-dimensional quantum many-body systems and provide an excellent starting point to address the fermionic BerezinskiiKosterlitz-Thouless transition with cold-atoms [14, 54].

We thank Igor Boettcher for useful discussions. This research was supported by the National Natural Science Foundation of China, Grant No. 11775123 (L.H.), National Key Research and Development Program of China, Grant No. 2018YFA0306503 (L.H.), and Australian Research Council's (ARC) Discovery Program, Grant No. FT140100003 (X.-J.L), Grant No. DP180102018 (X.J.L), and Grant No. DP170104008 (H.H.).

* These authors contributed equally to this work.

[1] M. Randeria, J.-M. Duan, and L.-Y. Shieh, Bound States, Cooper Pairing, and Bose Condensation in Two Dimensions, Phys. Rev. Lett. 62, 981 (1989).

[2] V. L. Berezinskii, Destruction of long-range order in onedimensional and two-dimensional systems with a continuous symmetry group. II. Quantum systems, Sov. Phys. JETP 34, 610 (1972) [Zh. Eksp. Teor. Fiz. 61, 1144 (1971)].

[3] J. M. Kosterlitz and D. J. Thouless, Ordering, metastability and phase transitions in two-dimensional systems, J. Phys. C 6, 1181 (1973).

[4] J. Levinsen and M. M. Parish, Strongly Interacting TwoDimensional Fermi Gases, in Annual Review of Cold Atoms and Molecules (World Scientific, Singapore, 2015), Volume 3, Chapter 1, Pages 1-75.

[5] A. V. Turlapov and M. Y. Kagan, Fermi-to-Bose crossover in a trapped quasi-2D gas of fermionic atoms, J. Phys.: Condens. Matter 29, 383004 (2017).

[6] V. Makhalov, K. Martiyanov, and A. Turlapov, GroundState Pressure of Quasi-2D Fermi and Bose Gases, Phys. Rev. Lett. 112, 045301 (2014).

[7] K. Martiyanov, T. Barmashova, V. Makhalov, and A. Turlapov, Pressure profiles of nonuniform twodimensional atomic Fermi gases, Phys. Rev. A 93, 063622 (2016).

[8] K. Fenech, P. Dyke, T. Peppler, M. G. Lingham, S. Hoinka, H. Hu, and C. J. Vale, Thermodynamics of an Attractive 2D Fermi Gas, Phys. Rev. Lett. 116, 045302 (2016).

[9] I. Boettcher, L. Bayha, D. Kedar, P. A. Murthy, M. Neidig, M. G. Ries, A. N. Wenz, G. Zürn, S. Jochim, and
T. Enss, Equation of State of Ultracold Fermions in the 2D BEC-BCS Crossover Region, Phys. Rev. Lett. 116, 045303 (2016).

[10] B. Fröhlich, M. Feld, E. Vogt, M. Koschorreck, W. Zwerger, and M. Köhl, Radio-Frequency Spectroscopy of a Strongly Interacting Two-Dimensional Fermi Gas, Phys. Rev. Lett. 106, 105301 (2011).

[11] A. T. Sommer, L. W. Cheuk, M. J. H. Ku, W. S. Bakr, and M. W. Zwierlein, Evolution of Fermion Pairing from Three to Two Dimensions, Phys. Rev. Lett. 108, 045302 (2012).

[12] Y. Zhang, W. Ong, I. Arakelyan, and J. E. Thomas, Polaron-to-Polaron Transitions in the Radio-Frequency Spectrum of a Quasi-Two-Dimensional Fermi Gas, Phys. Rev. Lett. 108, 235302 (2012).

[13] M. G. Ries, A. N. Wenz, G. Zürn, L. Bayha, I. Boettcher, D. Kedar, P. A. Murthy, N. Neidig, T. Lompe, and S. Jochim, Observation of Pair Condensation in the Quasi2D BEC- BCS Crossover, Phys. Rev. Lett. 114, 230401 (2015).

[14] P. A. Murthy, I. Boettcher, L. Bayha, M. Holzmann, D. Kedar, M. Neidig, M. G. Ries, A. N. Wenz, G. Zürn, and S. Jochim, Observation of the Berezinskii-Kosterlitz-Thouless Phase Transition in an Ultracold Fermi Gas, Phys. Rev. Lett. 115, 010401 (2015).

[15] E. Vogt, M. Feld, B. Fröhlich, D. Pertot, M. Koschorreck, and M. Köhl, Scale Invariance and Viscosity of a TwoDimensional Fermi Gas, Phys. Rev. Lett. 108, 070404 (2012).

[16] M. Holten, L. Bayha, A. C. Klein, P. A. Murthy, P. M. Preiss, and S. Jochim, Anomalous Breaking of Scale Invariance in a Two-Dimensional Fermi Gas, Phys. Rev. Lett. 121, 120401 (2018).

[17] T. Peppler, P. Dyke, M. Zamorano, S. Hoinka, and C. J. Vale, Quantum Anomaly and 2D-3D Crossover in Strongly Interacting Fermi Gases, Phys. Rev. Lett. 121, 120402 (2018).

[18] V. M. Loktev, R. M. Quick, and S. G. Sharapov, Phase fluctuations and pseudogap phenomena, Phys. Rep. 349, 1 (2001).

[19] M. Ruggeri, S. Moroni, and M. Boninsegni, Quasi-2D Liquid ${ }^{3}$ He, Phys. Rev. Lett. 111, 045303 (2013).

[20] H. Deng, H. Haug, and Y. Yamamoto, Exciton-polariton Bose-Einstein condensation, Rev. Mod. Phys. 82, 1489 (2010).

[21] J. A. Pons, D. Viganò, and N. Rea, A highly resistive layer within the crust of x-ray pulsars limits their spin periods, Nat. Phys. 9, 431 (2013).

[22] D. S. Petrov and G. V. Shlyapnikov, Interatomic collisions in a tightly confined Bose gas, Phys. Rev. A 64, 012706 (2001).

[23] G. Bertaina and S. Giorgini, BCS-BEC Crossover in a Two-Dimensional Fermi Gas, Phys. Rev. Lett. 106, 110403 (2011).

[24] A. A. Orel, P. Dyke, M. Delehaye, C. J. Vale, and H. $\mathrm{Hu}$, Density distribution of a trapped two-dimensional strongly interacting Fermi gas, New J. Phys. 13, 113032 (2011).

[25] J. Hofmann, Quantum Anomaly, Universal Relations, and Breathing Mode of a Two-Dimensional Fermi Gas, Phys. Rev. Lett. 108, 185303 (2012).

[26] E. Taylor and M. Randeria, Apparent Low-Energy Scale Invariance in Two-Dimensional Fermi Gases, Phys. Rev. Lett. 109, 135301 (2012). 
[27] M. Bauer, M. M. Parish, and T. Enss, Universal Equation of State and Pseudogap in the Two-Dimensional Fermi Gas, Phys. Rev. Lett. 112, 135302 (2014).

[28] M. Barth and J. Hofmann, Pairing effects in the nondegenerate limit of the two-dimensional Fermi gas, Phys. Rev. A 89, 013614 (2014).

[29] L. He, H. Lü, G. Cao, H. Hu, and X.-J. Liu, Quantum fluctuations in the BCS-BEC crossover of twodimensional Fermi gases, Phys. Rev. A 92, 023620 (2015).

[30] H. Shi, S. Chiesa, and S. Zhang, Ground-state properties of strongly interacting Fermi gases in two dimensions, Phys. Rev. A 92, 033603 (2015).

[31] B. C. Mulkerin, K. Fenech, P. Dyke, C. J. Vale, X.-J. $\mathrm{Liu}$, and $\mathrm{H}$. $\mathrm{Hu}$, Comparison of strong-coupling theories for a two-dimensional Fermi gas, Phys. Rev. A 92, 063636 (2015).

[32] E. R. Anderson and J. E. Drut, Pressure, Compressibility, and Contact of the Two-Dimensional Attractive Fermi Gas, Phys. Rev. Lett. 115, 115301 (2015).

[33] H. Hu, B. C. Mulkerin, U. Toniolo, L. He, and X.-J. Liu, Reduced Quantum Anomaly in a QuasiTwo-Dimensional Fermi Superfluid: Significance of the Confinement-Induced Effective Range of Interactions, Phys. Rev. Lett. 122, 070401 (2019).

[34] The definition of a $2 \mathrm{D}$ scattering length (denoted by $a_{2}$ ) used by Turlapov group in Ref. [6] and Ref. [7] is slightly different, and is related to $a_{2 D}\left(k_{0}\right)$ by, $a_{2 D}\left(k_{0}\right)=$ $\left(e^{\gamma_{E}} / 2\right) a_{2}$, where $\gamma_{E} \simeq 0.577$ is Euler's constant and $k_{0}=\sqrt{2 M \tilde{\mu}} / \hbar$. We also note that, in Ref. [33] we have used the notation $a_{2 D}=a_{2 D}(k \rightarrow 0)$, which is exactly $a_{s}$ defined in this work.

[35] S. K. Adhikari, Quantum scattering in two dimensions, Am. J. Phys. 54, 362 (1986).

[36] This equation can be rewritten as, $a_{z} / a_{3 D}=$ $\mathcal{F}\left[\varepsilon_{B} /\left(\hbar \omega_{z}\right)\right]$, where the function $\mathcal{F}(x)$ is given by, $\mathcal{F}(x)=\int_{0}^{\infty} d u\left(4 \pi u^{3}\right)^{-1 / 2}\left[1-e^{-x u} / \sqrt{\left(1-e^{-2 u}\right) /(2 u)}\right]$.

[37] X.-J. Liu and H. Hu, Self-consistent theory of atomic Fermi gases with a Feshbach resonance at the superfluid transition, Phys. Rev. A 72, 063613 (2005).

[38] L. M. Schonenberg, P. C. Verpoort, and G. J. Conduit, Effective-range dependence of two-dimensional Fermi gases, Phys. Rev. A 96, 023619 (2017).

[39] H. Hu, X.-J. Liu, and P. D. Drummond, Equation of state of a superfluid Fermi gas in the BCS-BEC crossover, Europhys. Lett. 74, 574 (2006).

[40] H. Hu, P. D. Drummond, and X.-J. Liu, Universal thermodynamics of strongly interacting Fermi gases, Nat. Phys. 3, 469 (2007).

[41] R. B. Diener, R. Sensarma, and M. Randeria, Quantum fluctuations in the superfluid state of the BCS-BEC crossover, Phys. Rev. A 77, 023626 (2008).

[42] See Supplemental Material at http://link.aps.org/supplemental/10.1103/.. for more information on the applicability of our effective theory, the choice of the $\beta$-function and the quantum anomaly in breathing mode frequency.
[43] The DMC simulations in Ref. 38] were carried out as a function of $k_{F}^{2} r_{\mathrm{eff}}^{2}=4 k_{F}^{2} R_{s} / \pi$ in the strongly interacting regime, where the mean-field solution for chemical potential $\mu_{\mathrm{MF}}$ is fixed to 0 . By solving the meanfield equation, we find the following relation between the effective range and $2 \mathrm{D}$ scattering length: $1 /\left(k_{F} a_{s}\right)=$ $\left[\left(\sqrt{1-4 k_{F}^{2} R_{s}}-1\right) /\left(-2 k_{F}^{2} R_{s}\right)\right]^{1 / 2}$. The green dot in Fig. 2 (b) corresponds to the DMC data at $k_{F}^{2} r_{\text {eff }}^{2}=-0.4$, leading to $k_{F}^{2} R_{s} \simeq-0.31416$ and $k_{F} a_{s} \simeq 1.1185$. This gives the ratio $R_{s} / a_{s}^{2} \simeq-0.2511$.

[44] D. A. Butts and D. S. Rokhsar, Trapped Fermi gases, Phys. Rev. A 55, 4346 (1997).

[45] We have used the peak chemical potential data $\tilde{\mu}_{0}=$ $\mu_{\text {peak }}+\varepsilon_{B} / 2$ listed in Table III of Supplemental Material of Ref. [9]. To have a robust procedure of measurement, in the experiment the chemical potential with the twobody binding energy subtracted $(\tilde{\mu})$ was assumed to be proportional to the local Fermi energy, $\tilde{\mu}=\varepsilon_{F} / c$ [9]. A ratio $1 / c$ was then obtained by fitting the linear relation at different densities and was supposed to provide the same information as $\mu_{\text {peak }}$ at the trap center. The ratio $1 / c$ is slightly smaller than $\tilde{\mu}_{0} / \varepsilon_{F}$, and is plotted in Fig. 1 of Ref. [9].

[46] Y.-Y. He, H. Shi, and S. Zhang, Reaching the Continuum Limit in Finite-Temperature Ab Initio Field-Theory Computations in Many-Fermion Systems, Phys. Rev. Lett. 123, 136402 (2019).

[47] D. S. Petrov, C. Salomon, and G. V. Shlyapnikov, Weakly Bound Dimers of Fermionic Atoms, Phys. Rev. Lett. 93, 090404 (2004).

[48] C. Menotti and S. Stringari, Collective oscillations of a one-dimensional trapped Bose-Einstein gas, Phys. Rev. A 66, 043610 (2002).

[49] H. Hu, G. Xianlong, and X.-J. Liu, Collective modes of a one-dimensional trapped atomic Bose gas at finite temperatures, Phys. Rev. A 90, 013622 (2014).

[50] L. P. Pitaevskii and A. Rosch, Breathing modes and hidden symmetry of trapped atoms in two dimensions, Phys. Rev. A 55, R853 (1997).

[51] M. Olshanii, H. Perrin, and V. Lorent, Example of a Quantum Anomaly in the Physics of Ultracold Gases, Phys. Rev. Lett. 105, 095302 (2010).

[52] We have calculated the density profile by using the AFQMC EoS and have then used the sum-rule equation (5) to determine the breathing mode frequency within AFQMC. This calculation improves the previous estimation given in Ref. [25] and Ref. [26].

[53] P. A. Murthy, N. Defenu, L. Bayha, M. Holten, P. M. Preiss, T. Enss, and S. Jochim, Quantum scale anomaly and spatial coherence in a 2D Fermi superfluid, Science 365, 268 (2019).

[54] B. C. Mulkerin, L. He, P. Dyke, C. J. Vale, X.-J. Liu, and $\mathrm{H}$. Hu, Superfluid density and critical velocity near the Berezinskii-Kosterlitz-Thouless transition in a twodimensional strongly interacting Fermi gas, Phys. Rev. A 96, 053608 (2017). 